\title{
Sprachlich-kommunikative Aspekte der medizinischen Indikation
}

\author{
Elsa Romfeld • Alice Schwab
}

(C) Der/die Autor(en) 2020

Die theoretische Auseinandersetzung mit der medizinischen Indikation als einem zentralen Begriff des ärztlichen Handelns ist in den letzten Jahren auch im deutschen Sprachraum verstärkt in den Fokus der Aufmerksamkeit gerückt; das belegen unter anderem die Zunahme an Publikationen ${ }^{1}$ sowie die Ausrichtung einschlägiger Tagungen ${ }^{2}$. Während dort die Explikation des Begriffs, die medizinischen Grundlagen oder die ärztliche Praxis, einschließlich rechtlicher Lage und ökonomischer Rahmenbedingungen, eine vergleichsweise intensive Betrachtung erfahren, werden die sprachlich-kommunikativen Aspekte zumeist, wenn überhaupt, nur am Rande beleuchtet. Dieser Themenschwerpunkt möchte genau diesen Perspektiven und Fragestellungen Raum geben. Wie zu zeigen ist, spielen neben medizinischwissenschaftlichen Entscheidungen kommunikativ vermittelte soziale Aspekte, beispielsweise Machthierachien, sowie sprachlich wirksame kulturelle Konzepte und Hintergrundannahmen eine wesentliche Rolle im Umgang mit der Indikation. Einführend soll im Folgenden zunächst die Problematik des Indikationsbegriffs bzw. der Indikationsstellung in der klinischen Praxis näher in den Blick genommen werden.

\footnotetext{
1 Beispiele dafür siehe unter „Literatur“ am Ende des Editorials.

2 Z. B. ,Medizinische Indikation und ökonomische Differenz“, Zentrum für Gesundheitsethik an der Evangelischen Akademie Loccum, 7.-8. November 2013; „Medizinische Indikation und ärztliche Verantwortung“, 3. Göttinger Symposium zum Medizinrecht, 27. Oktober 2017.
}

E. Romfeld, M.A. (ه)

Medizinische Fakultät Mannheim, Fachgebiet Geschichte, Theorie und Ethik der Medizin, Universität Heidelberg, Ludolf-Krehl-Straße 13-17, 68167 Mannheim, Deutschland

E-Mail: elsa.romfeld@medma.uni-heidelberg.de

Dr. med. A. Schwab, MAE

St. Franziskus Hospital Münster, Hohenzollernring 70, 48145 Münster, Deutschland

E-Mail: alice.schwab@sfh-muenster.de 


\section{Der Begriff der Indikation im klinischen Umfeld. Eine Problematisierung}

Im klinischen Sprachgebrauch ist der Begriff der Indikation allgegenwärtig. Er wird mit großer Selbstverständlichkeit verwendet, doch es fehlt eine klare Definition. Zwar existiert bei Ärzt*innen und Pflegekräften in der Regel eine Intuition, was damit gemeint sein könnte, sie bleibt aber vage. Eine Vielzahl allgemeiner Explikationen bietet zunächst das Internet an. Dort erläutern Wissensplattformen wie Wikipedia den Rezipient*innen etwa die absolute, relative, vitale oder selektive Indikation. In der ethischen Fachliteratur werden weitere Spezifizierungen vorgenommen: Neitzke (2015) spricht von der ärztlichen und medizinischen Indikation, Raspe (2015) von der generellen, individuellen und personalen Indikation, Wiesing (2017) beschreibt die Indikationsstellung, die Indikationsregel und das Indikationsgebiet. Parallel wird hinterfragt, ob und in welcher Form der Begriff der Indikation überhaupt Bestand haben sollte. So schlägt Marckmann (2015) vor, Indikation als „kryptonormativen Begriff“ durch „Wirksamkeit und Nutzen“ zu ersetzen. In der Summe dürften die verschiedenen Interpretationen und Ansätze unter den Kliniker*innen eher Verwirrung stiften als Klarheit schaffen.

Nicht nur der Begriff der Indikation selbst ist unscharf, es stellt sich zudem die Frage, wer darüber entscheidet, welche Maßnahme indiziert ist. So könnten es die Patient*innen sein, die ein Therapieziel erreichen möchten und damit den Rahmen der zu indizierenden Maßnahme festlegen. Alternativ sind es die Ärzt*innen, die aufgrund ihrer fachlichen Expertise einschätzen, welches Vorgehen indiziert ist. Lipp beschreibt den Prozess der Indikationsstellung folgendermaßen: „Sie muss zwar im Gespräch mit dem Patienten erfolgen [...], fällt aber letztlich in den Verantwortungsbereich des Arztes“ (Lipp 2015, S. 763). Die Bundesärztekammer wiederum sieht ausschließlich die Ärzt*innen in der Verantwortung. Als Entscheidungsgrundlagen für eine Indikation nennt sie objektive Parameter, ein moralisches Bewerten von Behandlungsbedarf und erstrebenswerten Zielen sowie die Individualität des Patienten. Wurde eine Indikation gestellt, so hat sie laut Bundesärztekammer ,normative Kraft“, „,normative Leitfunktion“ (BÄK 2015, S. 4). ${ }^{3}$ Die normativen Bedingungen für medizinisches Handeln wiederum sind in $\S 1$ (2) der (Muster-) Berufsordnung für die in Deutschland tätigen Ärztinnen und Ärzte (BÄK 2019a) festgelegt. So ist es ärztliche Aufgabe ,das Leben zu erhalten, die Gesundheit zu schützen und wiederherzustellen, Leiden zu lindern, Sterbenden Beistand zu leisten“. Für jede dieser Aufgaben gibt es verschiedenste Maßnahmen, die ,indiziert“ sein können. Für Patient*innen und Ärzt*innen bedeutet das, mit einem Begriff umgehen zu müssen, der nicht eindeutig definiert, jedoch gleichzeitig normativ wirksam ist. Diese Problematik soll an zwei Beispielen ${ }^{4}$ veranschaulicht werden; die konkreten Entscheidungssituationen illustrieren die Komplexität der Indikationsstellung.

\footnotetext{
3 Die Bundesärztekammer thematisiert die normative Leitfunktion der Indikation unter dem Aspekt der wunscherfüllenden Medizin und der damit einhergehenden Frage, ob Patient*innen nicht indizierte Maßnahmen wünschen können.

4 Die Fälle sind partiell konstruiert, gleichwohl realistisch.
} 
Beispiel 1 Der Patient Herr A leidet an einer schweren Arterienverkalkung der Beine. Von einer offenen Stelle am Unterschenkel breitet sich eine Blutvergiftung aus, die Antibiotikatherapie hat keine ausreichende Wirkung mehr. Die Amputation des Unterschenkels ist lebensrettend. Möchte Herr A leben, „muss“ die Amputation durchgeführt werden, die „Indikation steht““.

Meist akzeptieren Patient*innen die von den Ärzten gestellten Indikationen. Gelegentlich äußern sie Sätze wie „Die Operation muss ja gemacht werden“ oder „Es bleibt mir wohl nichts anderes übrig“. In diesen Formulierungen schwingt ein Gefühl des Ausgeliefertseins mit: Ist die Indikation einmal gestellt, ergeben sich Abläufe, die quasi schicksalhaft erscheinen. Selbst ein tödlicher Ausgang im Rahmen dieser Operation schiene durch die gestellte Indikation akzeptiert oder gar legitimiert.

Herr A entscheidet sich jedoch gegen die Amputation. Sein weit fortgeschrittenes Alter, schwere Begleiterkrankungen und die für ihn zu stark reduzierte Lebensqualität nach dem Eingriff geben den Ausschlag. Offiziell hat er das Recht, der Operation nicht zuzustimmen, seine Einwilligung zu verweigern. Sprachlichkommunikativ heißt das für ihn: Er muss „Nein“ sagen, er muss sich gegen eine gestellte Indikation und damit ggf. auch gegen die Person, die die Indikation gestellt hat, positionieren. Er muss seine Haltung erklären, die eigenen Wertvorstellungen darlegen und Argumente gegen diese Indikation anführen. Dies alles ist für ihn als direkt Betroffenen nicht leicht, insbesondere bei erhöhter Vulnerabilität vor dem Hintergrund seiner Krankheit, Verunsicherung und Ängste. Zudem wird er jetzt als „schwieriger Patient“ oder als „,nicht compliant“ bezeichnet, der ,,gegen ärztlichen Rat“ handelt und die Operation ,,verweigert“. Herr A wird möglicherweise sogar psychiatrisch vorgestellt, um seine Entscheidungs- bzw. Einwilligungsfähigkeit zu klären.

Selbstverständlich muss von ärztlicher Seite sichergestellt sein, dass die Patient*innen vollständig über den jeweils zu erwartenden Verlauf und die etwaigen Komplikationen informiert sind, die Situation einschätzen und verstehen können und damit einwilligungsfähig sind (BÄK 2019b). Doch ebenso hat jede*r Patient*in ein Recht auf eigene Wertvorstellungen, Achtung der Autonomie, sogar auf - vermeintlich oder tatsächlich - unvernünftige Entscheidungen. Bemerkenswert ist: Der Fall, dass Patient*innen einer indizierten Maßnahme zustimmen, führt nicht dazu, ihre Entscheidungsfähigkeit anzuzweifeln, selbst wenn der Eingriff mit einem hohen Risiko für Komplikationen oder mit dem des Versterbens verbunden ist. Sind Patient*innen aber nicht bereit, die aufgrund einer Symptomatik angeblich eindeutig indizierte Maßnahmen durchführen zu lassen, stören sie das funktionierende System: Abläufe müssen verändert, das Therapieziel neu geklärt, andere indizierte Maßnahmen gefunden werden. Patient*innen können daher in bestimmten Situationen praktisch nur schwer eine indizierte Maßnahme ablehnen. Mit der Indikationsstellung wird eine Entscheidung getroffen, ein Fahrplan festgelegt für den weiteren diagnostischen oder therapeutischen Weg. Je schwerer das Krankheitsbild, je bedrohlicher der potentielle Verlauf einer Erkrankung, umso nachdrücklicher scheint dabei der Charakter des Indikationsbegriffs zu werden. Bis zur Indikationsstellung hingegen ist Verhandlungsspielraum über Therapieziele oder Wünsche des Patienten. Umso wichtiger ist es, dass Ärzt*innen in ihre Indikationsstellung hier frühzeitig möglichst viele Aspekte einfließen lassen, die für Patient*innen relevant sein können. 
Beispiel 2 Die Patientin Frau B hat sich bei einem Sturz eine Schenkelhalsfraktur zugezogen. Darüber hinaus leidet sie an einem weit fortgeschrittenen bösartigen Tumor, hat viel an Gewicht verloren und ist sehr schwach. Die behandelnde Ärztin schätzt die Lebenserwartung von Frau B aufgrund ihres stark reduzierten Allgemeinzustandes als sehr kurz ein. Sie zieht nun die aktuelle AWMF-Leitlinie zur Schenkelhalsfraktur des Erwachsenen (Bonnaire und Weber 2015) zurate. Dort heißt es:

- „Die Abklärung sollte die operative Behandlung nicht verzögern“ (Bonnaire und Weber 2015, S. 16).

- „Die operative Behandlung ist das Verfahren der Wahl bei der Behandlung der Schenkelhalsfraktur“ (Bonnaire und Weber 2015, S. 19).

- „Patienten mit Schenkelhalsfraktur sollten so schnell wie möglich innerhalb von $24 \mathrm{~h}$ operiert werden, wenn der Allgemeinzustand des Patienten dies zulässt“* (Bonnaire und Weber 2015, S. 26).

Die Bezeichnung der Operation als „Verfahren der Wahl“ und der normative Charakter der Formulierungen lassen der Ärztin kaum eine Alternative. Kann es anhand dieser Vorgaben noch eine andere Indikation geben als die Operation? In der Leitlinie heißt es immerhin auch: „Bei Patienten mit der Kombination von ausgeprägtem Herzversagen, Bronchopneumonie und signifikanter Malnutrition sollte die operative Therapie zurückhaltend erwogen werden, da ein hoher Anteil die perioperative Phase nicht überlebt" (Bonnaire und Weber 2015, S. 18). Diese Formulierung ist für die Ärztin in der konkreten Situation allerdings nicht hinreichend hilfreich, denn auch ein ,zurückhaltendes Erwägen“ der Operation schließt die Indikation zur Operation nicht aus.

Es ist nun folgendes Dilemma entstanden: Die Ärztin steht unter dem Druck, eine Indikation stellen zu müssen - Operation oder konservative Therapie. Die Operation ist indiziert, da die Fraktur stabilisiert und die daraus entstehenden Schmerzen durch eine Operation reduziert werden können. Und: Die Operation ist nicht indiziert, da die Gesamtsituation der Patientin (aus Sicht der Ärztin) so schlecht ist, dass sie die perioperative Phase möglicherweise nicht überleben wird. Sie befürchtet, die Patientin könnte aufgrund der fortgeschrittenen Tumorerkrankung und ihrer Schwäche durch die Operation für einige Tage sehr beeinträchtigt sein oder gar perioperativ versterben. Sie stellt sich die Frage, ob die weit fortgeschrittene Tumorerkrankung der Patientin ausreicht, um die Operation als nicht indiziertes Verfahren gar nicht erst anzubieten. Sie würde damit ihre eigene Einschätzung über einen allgemeinen Konsens stellen und der Patientin ein Standardverfahren vorenthalten, weil sie den Nutzen der Operation geringer einschätzt als den Schaden für die Patientin. Sie würde - aus ihrer Sicht zum Wohl der Patientin - nicht ,alles“ tun, sondern bewusst auf die Operation verzichten und ein alternatives Vorgehen wählen, hier: die Indikation zur konservativen Therapie stellen.

Was aber, wenn die Patientin von sich aus eine Operation wünschte, weil sie sehr starke Schmerzen hat, die nur mit starken Schmerzmitteln und den daraus resultierenden Nebenwirkungen wie Benommenheit und Müdigkeit zu beherrschen sind? Bereits jetzt spürt Frau B diese Nebenwirkungen, die für sie nicht akzeptabel sind. Sie möchte die ihr verbleibende Zeit selbstbestimmt nutzen können und könnte 
ihr vorzeitiges Versterben eher akzeptieren als eine Einschränkung ihrer geistigen Leistungskraft. Die Patientin hat nun ein Therapieziel festgelegt, das nur mit einer Operation zu erreichen ist. Ist die Operation also jetzt eher indiziert?

An beiden Beispielen wird deutlich: Der Begriff der Indikation unterstellt eine Sicherheit der Entscheidung, die in vielen Situationen so nicht gegeben ist. Gerade in komplizierten gesundheitlichen Konstellationen ist der Rückgriff auf Standardverfahren, also auf zwingend umzusetzende Indikationen auf Grundlage objektiver Parameter, nicht möglich. Es bleibt eine Abwägung des Therapieziels, des Schadens und des Nutzens einzelner medizinischer Maßnahmen. Dies kann nur individuell im gemeinsamen Gespräch erfolgen, um die verschiedenen medizinischen Optionen hinsichtlich der Werte und Ziele der Patient*innen zu beurteilen. Ärzt*innen können im Sinne des informed consent ihren Patient*innen lediglich eine Empfehlung für oder gegen ein Verfahren geben. Sätze wie „es ist indiziert“ oder „die Indikation steht" aber suggerieren Objektivität. Sie verführen durch ihre subtile Normativität zu einem ungewollten Paternalismus, der heute anachronistisch anmutet. Ob eine die Autonomie achtende, wertschätzende Gesprächskultur sich mit alternativen Begriffen oder Konzepten, die eventuell weniger normative und imperative Konnotationen als „Indikation“ haben, besser bewerkstelligen lässt und welche das im Einzelnen sein könnten, muss an dieser Stelle offen bleiben.

In den drei folgenden Beiträgen zum Themenschwerpunkt wird das Phänomen der medizinischen Indikation in seiner Mehrdimensionalität, speziell in seiner sprachlich sich manifestierenden Konzeptbeladenheit und Interpretationssensibilität exemplarisch ausgeleuchtet, indem die Autor*innen an konkreten Beispielen Voraussetzungen für die Indikationsstellung explizieren und reflektieren und auf diese Weise Konsequenzen des Indikationsbegriffs für die medizinische Praxis aufzeigen.

\section{Die Beiträge im Einzelnen}

Im ersten Beitrag untersucht Nadia Primc die sprachlich vermittelten Interpretationsrahmen (,framings“), in welche die sechsmonatige Karenzregel bei der Lebertransplantation jeweils gestellt wird. Die Karenzregel fordert von Patient*innen mit einer alkoholinduzierten Lebererkrankung eine sechsmonatige Karenzzeit, bevor diese auf die Warteliste für eine Lebertransplantation aufgenommen werden können. In dem Beitrag werden vier unterschiedliche „framings“ der Karenzregel identifiziert, wobei die ersten drei wiederum die Frage nach der medizinischen Indiziertheit der Karenzforderung aufwerfen, die im Beitrag hinsichtlich ihrer ethischen Fundierung näher untersucht wird.

Im Anschluss widmet sich Giovanni Rubeis in seinem Beitrag unter der Überschrift „Gute Elternschaft“ den normativen Urteilen, die hinter dem Konzept der Indikation in der Reproduktionsmedizin stehen. Der übliche Sprachgebrauch suggeriert, dass die Indikation rein auf empirisch-klinischen Fakten basiert. Dabei wird ein soziokulturelles Skript verdeckt, das den normativen Gehalt der Indikation in der Reproduktionsmedizin darstellt. Dieses Skript enthält gesellschaftliche Anschauungen, Normen und Rollenbilder und kann als ,gute Elternschaft“ bezeichnet werden. Somit fließen in die Indikationsstellung in der Reproduktionsmedizin nicht allein 
klinische Fakten ein, sondern auch normative Urteile darüber, wer zur Elternschaft berechtigt ist.

Abschließend wird im Beitrag von Katharina Fürholzer das komplexe Zusammenspiel von Sprache und Indikation anhand der Textsorte des medizinischen Fallberichts analysiert. Ausgangspunkt ist die Beobachtung, dass die Stimme von Patienten im wissenschaftlichen Kommunikationsraum - anders als in der klinischen Patientenversorgung - bislang nicht ausreichend Gehör findet. Vor diesem Hintergrund wird im Beitrag die spezifische Bedeutung subjektiver Patientenperspektiven und -erzählungen für die von (vermeintlicher) Objektivität geprägte Textsorte des Fallberichts und aus ihr hervorgehende Indikationsstellungen diskutiert.

Danksagung Wir danken den Autor*innen für ihre Beiträge und die fruchtbare Zusammenarbeit sowie den externen Gutachter*innen für ihre konstruktiven Rückmeldungen. Der Schriftleitung von Ethik in der Medizin danken wir, dass dieser Themenschwerpunkt ermöglicht wurde; ein besonderer Dank gilt Frau Kähling für ihre freundliche und zuverlässige Unterstützung.

Funding Open Access funding provided by Projekt DEAL.

Open Access Dieser Artikel wird unter der Creative Commons Namensnennung 4.0 International Lizenz veröffentlicht, welche die Nutzung, Vervielfältigung, Bearbeitung, Verbreitung und Wiedergabe in jeglichem Medium und Format erlaubt, sofern Sie den/die ursprünglichen Autor(en) und die Quelle ordnungsgemäß nennen, einen Link zur Creative Commons Lizenz beifügen und angeben, ob Änderungen vorgenommen wurden.

Die in diesem Artikel enthaltenen Bilder und sonstiges Drittmaterial unterliegen ebenfalls der genannten Creative Commons Lizenz, sofern sich aus der Abbildungslegende nichts anderes ergibt. Sofern das betreffende Material nicht unter der genannten Creative Commons Lizenz steht und die betreffende Handlung nicht nach gesetzlichen Vorschriften erlaubt ist, ist für die oben aufgeführten Weiterverwendungen des Materials die Einwilligung des jeweiligen Rechteinhabers einzuholen.

Weitere Details zur Lizenz entnehmen Sie bitte der Lizenzinformation auf http://creativecommons.org/ licenses/by/4.0/deed.de.

Interessenkonflikt E. Romfeld und A. Schwab geben an, dass kein Interessenkonflikt besteht.

\section{Literatur}

Bonnaire F, Weber A (2015) Schenkelhalsfraktur des Erwachsenen, AWMF-Leitlinie. https://www.awmf. org/uploads/tx_szleitlinien/012-0011_S2e_Schenkelhalsfraktur_2015-10_01.pdf. Zugegriffen: 28. Juli 2020

Bundesärztekammer (BÄK) (2015) Stellungnahme „Medizinische Indikationsstellung und Ökonomisierung“. Dtsch Arztebl 112(18):A-836/B-708/C-684

Bundesärztekammer (BÄK) (2019a) Bekanntmachung (Muster-)Berufsordnung für die in Deutschland tätigen Ärztinnen und Ärzte - MBO-Ä 1997 -*) in der Fassung der Beschlüsse des 121. Deutschen Ärztetages 2018 in Erfurt geändert durch Beschluss des Vorstandes der Bundesärztekammer am 14.12.2018. Dtsch Arztebl. https://doi.org/10.3238/arztebl.2019.mbo_daet2018b

Bundesärztekammer (BÄK) (2019b) Hinweise und Empfehlungen der Bundeärztekammer zum Umgang mit Zweifeln an der Einwilligungsfähigkeit bei erwachsenen Patienten. Dtsch Arztebl 116(22):A1133/B-933/C-921

Lipp V (2015) Die medizinische Indikation - ein „Kernstück ärztlicher Legitimation“? MedR 33:762-766. https://doi.org/10.1007/s00350-015-4126-8

Marckmann G (2015) Wirksamkeit und Nutzen als alternative Konzepte zur medizinischen Indikation. In: Dörries A, Lipp V (Hrsg) Medizinische Indikation. Kohlhammer, Stuttgart, S 113-124

Neitzke G (2015) Medizinische und ärztliche Indikation - zum Prozess der Indikationsstellung. In: Dörries A, Lipp V (Hrsg) Medizinische Indikation. Kohlhammer, Stuttgart, S 83-93 
Raspe H (2015) Die medizinische Indikation und ihre Regulierung in Zeiten der evidenzbasierten Medizin. In: Dörries A, Lipp V (Hrsg) Medizinische Indikation. Kohlhammer, Stuttgart, S 94-112

Wiesing U (2017) Indikation. Theoretische Grundlagen und Konsequenzen für die ärztliche Praxis. Kohlhammer, Stuttgart 